\title{
Low-dose menaquinone-7 supplementation improved extra-hepatic vitamin $K$ status, but had no effect on thrombin generation in healthy subjects
}

\author{
Elke Theuwissen $^{1 *}$, Ellen C. Cranenburg ${ }^{1}$, Marjo H. Knapen ${ }^{1}$, Elke J. Magdeleyns ${ }^{1}$, Kirsten J. Teunissen ${ }^{1}$, \\ Leon J. Schurgers ${ }^{1,2}$, Egbert Smit ${ }^{1}$ and Cees Vermeer ${ }^{1}$ \\ ${ }^{1}$ VitaK \& Cardiovascular Research Institute Maastricht (CARIM), Maastricht University, Oxfordlaan 70,6229 EV Maastricht, \\ The Netherlands \\ ${ }^{2}$ Cardiovascular Research Institute Maastricht (CARIM), Maastricht University, Universiteissingel 50, 6229 ER Maastricht, \\ The Netherlands
}

(Submitted 28 September 2011 - Final revision received 25 November 2011 - Accepted 28 November 2011 - First published online 31 January 2012)

\begin{abstract}
Vitamin $\mathrm{K}$ is required for the carboxylation of Gla-proteins in the liver (coagulation factors) and extra-hepatic tissues, such as bone (osteocalcin, OC), and arterial wall (matrix Gla-protein, MGP). Although the coagulation factors are essentially fully carboxylated under normal conditions, 10-40\% of OC and MGP remains undercarboxylated. We were therefore interested to study the dose-response effects of extra intake of menaquinones on the carboxylation of the extra-hepatic Gla-proteins. A total of forty-two healthy Dutch men and women aged between 18 and 45 years were randomised into seven groups to receive: placebo capsules or menaquinone-7 (MK-7) capsules at a daily dose of 10, 20, 45, 90, 180 or $360 \mu \mathrm{g}$. Circulating uncarboxylated OC (ucOC), carboxylated OC (cOC) and desphospho-uncarboxylated MGP were measured by ELISA. The ucOC: $\mathrm{COC}$ ratio was calculated from circulating ucOC and cOC values. Endogenous thrombin potential and peak height were determined by calibrated automated thrombography. To increase the statistical power, we collapsed the treatment groups into three dosage groups: placebo, low-dose supplementation (doses below RDA, Commission Directive 2008/100/EC), and highdose supplementation (doses around RDA, Commission Directive 2008/100/EC). MK-7 supplementation at doses in the order of the RDA (Commission Directive 2008/100/EC) increased the carboxylation of circulating OC and MGP. No adverse effects on thrombin generation were observed. Extra MK-7 intake at nutritional doses around the RDA (Commission Directive 2008/100/EC) improved the carboxylation of the extra-hepatic vitamin K-dependent proteins. Whether this improvement contributes to public health, i.e. increasing the protection against age-related diseases needs further investigation in specifically designed intervention trials.
\end{abstract}

Key words: Matrix Gla-protein: Osteocalcin: Menaquinone-7: Endogenous thrombin potential

Overall, seventeen vitamin K-dependent proteins, also known as Gla-proteins, have been identified to date. Of these, one group of Gla-proteins is involved in haemostasis and includes clotting factors II, VII, IX, $\mathrm{X}$ and proteins $\mathrm{C}, \mathrm{S}, \mathrm{Z}^{(1)}$. These proteins are almost exclusively synthesised by the liver; with the exception of protein S. Mutant mice revealed that endothelial cells synthesise about $45 \%$ of circulating protein $S^{(2)}$. The extra-hepatic group is formed among others by Gla-proteins involved in extracellular matrix mineralisation, e.g. osteocalcin (OC; bone), matrix Gla-protein (MGP; cartilage and arterial vessel wall) and periostin (bone).

Whereas incompletely carboxylated coagulation factors are seldom seen in healthy subjects, substantial fractions of OC and MGP circulate in their uncarboxylated forms, suggesting a suboptimal vitamin $\mathrm{K}$ status in extra-hepatic tissues. Elevated concentrations of uncarboxylated OC (ucOC) have been associated with osteoporotic fractures ${ }^{(3)}$ and high circulating concentrations of uncarboxylated MGP (ucMGP) have been shown to predict CVD mortality ${ }^{(4)}$. Inadequate menaquinone intake has also been associated with increased risk of cancer $^{(5)}$. The triage theory postulates that long-term vitamin insufficiency is a risk factor for the development of age-related diseases $^{(6)}$. In most cases, risk reduction of these diseases requires vitamin intakes well above the present RDA (RDA = $75 \mu \mathrm{g}$; Commission Directive 2008/100/EC; Commission of the European Communities, 2008).

We previously demonstrated that menaquinone-7 (MK-7) supplementation at a low dose, but above the RDA (Commission Directive 2008/100/EC), mediates OC carboxylation with substantially higher efficacy than phylloquinone ${ }^{(7)}$. Here, we

\footnotetext{
Abbreviations: CAT, calibrated automated thrombography; cOC, carboxylated osteocalcin; dp-ucMGP, desphospho-uncarboxylated matrix Gla-protein; ETP, endogenous thrombin potential; MGP, matrix Gla-protein; MK-7, menaquinone-7; NP, normal plasma; OC, osteocalcin; PH, peak height; TG, thrombin generation; ucOC, uncarboxylated osteocalcin.
}

*Corresponding author: Dr E. Theuwissen, fax +31 433885889, email e.theuwissen@vitak.com 
hypothesised that increased carboxylation of OC and MGP can be obtained with MK-7 administration at nutritional doses. OC helps in structuring bone by restricting calcification within the bone fibrils and MGP is the most important local inhibitor of vascular calcification; a diet leading to increased carboxylation of both proteins may contribute to public health. Since the key role of vitamin $\mathrm{K}$ in regulating haemostasis is generally known, an obvious question is whether increased vitamin $\mathrm{K}$ intake may lead to increased thrombosis tendency. Whereas this is certainly true for subjects receiving vitamin $\mathrm{K}$ antagonists, there is no evidence for vitamin K-induced hypercoagulability in healthy subjects. The recent development of calibrated automated thrombography (CAT) enabled us to measure the effects on thrombin generation (TG). The objectives of the present study were therefore to study the dose-response effects of extra MK-7 supplementation on the carboxylation of OC and MGP and the effects on TG as an indicator of safety.

\section{Experimental methods}

\section{Subjects}

Healthy men and women aged between 18 and 45 years were recruited from the Maastricht University community (Maastricht, The Netherlands) through mailings and small advertisements. Exclusion criteria were BMI $>30 \mathrm{~kg} / \mathrm{m}^{2}$, coagulation disorders, metabolic or gastrointestinal diseases, chronic diseases (diabetes mellitus, CVD, cancer), the use of supplements containing vitamin $\mathrm{K}$, routine medication that interferes with vitamin $\mathrm{K}$ and/or coagulation metabolism, participation in a clinical study 3 months before this study, and soya allergy. Based on these exclusion criteria and a previous health check (based on interviews and questionnaires), forty-two volunteers were selected for this intervention study. There were no participant dropouts during the period of the study.

This study was conducted according to the guidelines laid down in the Declaration of Helsinki and all procedures involving human subjects were approved by the Medical Ethics Committee of Maastricht University Medical Centre (Maastricht, The Netherlands). Written informed consent was obtained from all subjects before entering the study. Trial registration code: clinicaltrials.gov NCT00483431.

\section{Study design}

This study was designed as an exploratory pilot study to estimate the effective dose of MK-7 (to improve carboxylation of both OC and MGP) for a more elaborate clinical trial to investigate the effect of MK-7 supplementation on clinical endpoints, including bone mineral density, bone mineral content and vascular characteristics.

The study had a double-blind, randomised, controlled design. Participants were randomised into seven groups to receive one of the following supplements (capsules): placebo or MK-7 at a daily dose of $10,20,45,90,180$ or $360 \mu \mathrm{g}$. The supplements were taken once daily with either breakfast or dinner during a period of 12 weeks. During the intervention period, participants returned to the VitaK BV laboratories at days $0,1,3,7,14,28,42,56,70$ and 84 for blood sampling.

During the study period, subjects were asked to consume no more than normal amounts of green vegetables $(<200 \mathrm{~g} / \mathrm{d}$ of spinach, broccoli, Brussels sprouts and green cabbages) and (curd) cheese $(<50 \mathrm{~g} / \mathrm{d})$, and no natto during the study. The participants were also asked to refrain from consuming these food items and alcohol for at least $24 \mathrm{~h}$ before blood sampling. Compliance was measured by capsule counts at the end of the intervention period; the mean compliance for the MK-7 supplement was $92 \%$. Any noticeable changes in health, dietary pattern, physical activity and/or medication use were recorded.

\section{Study products}

The capsules were produced in four different MK-7 (MenaQ7) dosages $(0,10,45$ and $360 \mu \mathrm{g} /$ capsule) by NattoPharma ASA and delivered directly to VitaK BV. The capsules contained linseed oil, natto oil, potato starch, sunflower oil, carnauba wax (coating) and ferric oxide (colouring agent). Capsules with $0,10,45$ and $360 \mu \mathrm{g}$ of MK-7 contained $0,7,30$ and $240 \mathrm{mg}$ of natto oil and 240,233, 210 and $0 \mathrm{mg}$ of linseed oil, respectively. All other ingredients were equal for the different MK-7 capsules.

\section{Blood sampling}

Fasting venous blood was collected at days 0, 1, 3, 7, 14, 28, 42, 56, 70 and 84 for the preparation of serum and citrate plasma (Greiner Bio-One BV). At days 0 and $84,10 \mathrm{ml}$ of venous blood was collected for the preparation of plateletpoor plasma. For serum preparation, blood $(10 \mathrm{ml})$ was allowed to clot for $30 \mathrm{~min}$ at room temperature, centrifuged at $3000 \boldsymbol{g}$ for $15 \mathrm{~min}$, aliquoted and stored at $-80^{\circ} \mathrm{C}$. Plasma was prepared immediately after blood sampling $(10 \mathrm{ml})$ by centrifugation at $3000 \mathrm{~g}$ for $15 \mathrm{~min}$. Also, platelet-poor plasma was prepared after sampling $(10 \mathrm{ml})$ by a two-step centrifugation: (1) $3000 \boldsymbol{g}$ for $15 \mathrm{~min}$ and (2) $10000 \boldsymbol{g}$ for $10 \mathrm{~min}$. Plasma was aliquoted and partly stored at $-80^{\circ} \mathrm{C}$. Serum and plasma samples were stored at $-80^{\circ} \mathrm{C}$ until analysis. All analyses were performed in duplicate (except for vitamin $\mathrm{K}$ analyses), and mean values are given throughout the present paper.

\section{Vitamin $K$}

Circulating phylloquinone and MK-7 concentrations were measured at the end of the intervention period. Compliance was also checked by means of circulating MK-7. Plasma phylloquinone and MK-7 concentrations were assayed using $\operatorname{HPLC}^{(8)}$.

\section{Extra-hepatic Gla-proteins}

Serum ucOC and carboxylated OC (cOC) concentrations were determined by separate commercial dual-antibody ELISA tests (Takara Shuzo Company Limited). An in-house control pool 
was run on all ELISA plates. The ucOC:COC ratio was calculated from circulating ucOC and cOC values, and used as a marker for vitamin $\mathrm{K}$ status in bone. Time-point samples of one subject were analysed on the same ELISA plate. The intra- and inter-assay variations for ucOC were 4.8 and $7.7 \%$, respectively. For cOC, these variations were 2.3 and $10 \cdot 6 \%$, respectively.

Plasma desphospho-uncarboxylated MGP (dp-ucMGP) was measured by an in-house dual-antibody ELISA test ${ }^{(9)}$. Briefly, the capture antibody specifically recognising dp-MGP is bound to the microtitre plate. After incubation with the sample, a biotinylated conformation-specific antibody against ucMGP is used as a second detecting antibody. Staining was performed with streptavidine using 3,3',5,5'-tetramethylbenzidine as a substrate. An in-house control pool was run on all ELISA plates. Time-point samples of one subject were analysed on the same ELISA plate. The intra- and inter-assay variations were 5.6 and $9.9 \%$, respectively.

\section{Thrombin generation}

TG in tissue factor-triggered platelet-poor plasma was measured by means of CAT (Thrombinoscope BV), which employs a low-affinity fluorogenic substrate to continuously monitor thrombin activity in clotting plasma ${ }^{(10)}$. Fluorescence was read with a Fluoroskan Ascent reader (Thermo Labsystems) using a 390/460 filter set. TG curves were calculated with the Thrombinoscope software (Thrombinoscope BV).

Following this, two parameters were derived from the TG curves: endogenous thrombin potential (ETP, area under the TG curve) and thrombin peak height (PH). Each TG measurement included pooled normal plasma (NP), and both ETP and $\mathrm{PH}$ are expressed as the ratio of a patient value to the $\mathrm{NP}$ value; value $_{\text {test }} /$ value $_{\mathrm{NP}}$. Pooled NP was prepared at the Departments of Hematology and Clinical Chemistry of the Academic Hospital Maastricht (AZM) by pooling plasma from eighty-five healthy volunteers who did not use medication. The intra- and inter-assay variations were $<10 \%$.

\section{Statistical analyses}

At baseline, sex differences were tested by the independentsamples $t$ test $(P<0.05$ was considered statistically significant). Data are presented as means with standard deviations. Because of small group sizes, the non-parametric MannWhitney test was used to study differences in circulating vitamin $\mathrm{K}$ concentrations after 3-month supplementation. End values were compared to the end value of the placebo group after adjustment for multiple comparisons $(P<0.008$ was considered statistically significant). Data are presented as medians with ranges. A paired samples $t$ test (baseline values as reference values) was used to study the effects of MK-7 supplementation on ETP and PH values (comparison between baseline and end values). Correlation analysis was performed with the Pearson test $(P<0.05$ was considered statistically significant).

To increase the statistical power, we collapsed the seven treatment groups into three dosage groups: placebo, low-dose MK-7 (doses below the RDA, Commission Directive 2008/100/EC) and high-dose MK-7 (doses around the RDA, Commission Directive 2008/100/EC). Associations between extra MK-7 intake and the end value of the measure of interest (ucOC, cOC, ucOC:COC, dp-ucMGP) were investigated with ANCOVA including sex as a fixed factor and the following variables as covariates: baseline value, age and BMI. Only the main effects were tested, since the interaction terms with the measure of interest were not significant. Multiple comparisons were made using Bonferroni adjustment $(P<0.05$ was considered statistically significant). Baseline and end values are presented as medians with ranges for the originally designed treatment groups (seven groups). Mean percentage changes as compared to baseline are illustrated for the compiled groups: placebo, low-dose MK-7 and high-dose MK-7.

Statistical analyses were performed using SPSS for Windows, version 15 (SPSS, Inc.).

\section{Results}

\section{Baseline characteristics}

As summarised in Table 1, baseline characteristics were similar for men and women, with the exception of higher body weight $(P<0.001)$ and higher $\mathrm{COC}$ concentrations $(P=0.005)$ in men. Both ETP $(P=0.008)$ and PH $(P=0.001)$ values were lower in men than in women. A total of eighteen women were using oral contraceptives, and none of the participants smoked.

\section{Vitamin $K$}

Plasma phylloquinone concentrations were within the normal range $(0 \cdot 2-3 \cdot 2 \mathrm{ng} / \mathrm{ml})$ and did not differ between the intervention groups (Table 2). Plasma MK-7 concentrations increased with increasing amount of MK-7 supplementation, indicating compliance to the intervention. As compared to the placebo group, the increase in circulating concentrations became significant from an intake of $90 \mu \mathrm{g} / \mathrm{d}$.

Table 1. Baseline characteristics†

(Mean values and standard deviations)

\begin{tabular}{|c|c|c|c|c|c|c|}
\hline & \multicolumn{2}{|c|}{ All $(n$ 42) } & \multicolumn{2}{|c|}{ Men $(n 20)$} & \multicolumn{2}{|c|}{ Women (n 22) } \\
\hline & Mean & SD & Mean & SD & Mean & SD \\
\hline \multicolumn{7}{|l|}{ Anthropometric data } \\
\hline Age (years) & 28 & 7 & 28 & 7 & 28 & 7 \\
\hline Weight (kg) & 74 & 12 & 81 & 11 & $68^{*}$ & 7 \\
\hline $\mathrm{BMI}\left(\mathrm{kg} / \mathrm{m}^{2}\right)$ & 24 & 3 & 24 & 3 & 23 & 3 \\
\hline \multicolumn{7}{|l|}{ Biochemical markers } \\
\hline ucOC (ng/ml) & $4 \cdot 11$ & $2 \cdot 71$ & 4.44 & 3.27 & 3.82 & $2 \cdot 13$ \\
\hline $\operatorname{cOC}(\mathrm{ng} / \mathrm{ml})$ & 5.59 & $2 \cdot 39$ & $6 \cdot 64$ & $2 \cdot 65$ & $4 \cdot 64^{*}$ & 1.68 \\
\hline ucOC:cOC ratio & 0.82 & 0.54 & 0.67 & 0.39 & 0.95 & 0.63 \\
\hline dp-ucMGP (pmol/l) & 429 & 140 & 432 & 113 & 427 & 164 \\
\hline $\operatorname{ETP}(\mathrm{nmol} / \mathrm{l} \times \mathrm{min})$ & 1516 & 366 & 1362 & 331 & $1655^{\star}$ & 346 \\
\hline $\mathrm{PH}(\mathrm{nmol} / \mathrm{l})$ & 333 & 85 & 291 & 83 & $372^{\star}$ & 67 \\
\hline
\end{tabular}

ucOC, uncarboxylated osteocalcin; cOC, carboxylated osteocalcin; dp-ucMGP, desphospho-uncarboxylated matrix Gla-protein; ETP, endogenous thrombin potential; $\mathrm{PH}$, peak height.

${ }^{*}$ Mean values were significantly different $(P<0.05)$

$\dagger$ Sex differences were tested by the independent-samples $t$ test. 
Table 2. Circulating vitamin $\mathrm{K}$ concentrations after 3-month supplementation†

(Medians and ranges)

\begin{tabular}{|c|c|c|c|c|c|}
\hline & \multirow{2}{*}{$\begin{array}{c}\text { MK-7 } \\
\text { dose } \\
(\mu \mathrm{g} / \mathrm{d})\end{array}$} & \multicolumn{2}{|c|}{ Phylloquinone (ng/ml) } & \multicolumn{2}{|c|}{ MK-7 (ng/ml) } \\
\hline & & Median & Range & Median & Range \\
\hline Group 1 & 0 & 0.2 & $0.0-0.5$ & 0.4 & $0.0-0.5$ \\
\hline Group 2 & 10 & 0.8 & $0.3-1.3$ & 0.6 & $0.4-2.1$ \\
\hline Group 3 & 20 & 0.5 & $0.1-1.0$ & 0.5 & $0.0-1.6$ \\
\hline Group 4 & 45 & 0.3 & $0 \cdot 1-1 \cdot 2$ & 1.4 & $0.4-4.6$ \\
\hline Group 5 & 90 & 0.2 & $0.1-0.9$ & $1.5^{\star}$ & $0.5-4.2$ \\
\hline Group 6 & 180 & 0.2 & $0.1-0.4$ & $3 \cdot 4^{*}$ & $1 \cdot 1-6 \cdot 5$ \\
\hline Group 7 & 360 & 0.4 & $0.3-0.8$ & $5 \cdot 5^{\star}$ & $4 \cdot 1-10 \cdot 6$ \\
\hline
\end{tabular}

MK-7, menaquinone-7.

${ }^{*} P<0.008$ for multiple comparisons.

†A total of forty-two participants were randomised into seven groups (six subjects/group) to receive one of the following supplements (capsules): placebo or MK-7 at a daily dose of $10,20,45,90,180$ or $360 \mu$ g. Circulating phylloquinone and MK-7 concentrations were measured at the end of the intervention period. Between-group differences (differences as compared to placebo) were tested by the non-parametric Mann-Whitney test.

\section{Osteocalcin and matrix Gla-protein}

Circulating OC and MGP values at baseline and after 3-month supplementation are described in Table 3. As compared to the placebo group, MK-7 supplementation at doses around the RDA (Commission Directive 2008/100/EC; doses described as high-dose MK-7) significantly improved the carboxylation of OC and MGP (Fig. 1); significant decreases were seen in circulating ucOC and dp-ucMGP levels as well as in the ucOC: supplementation

(Medians and ranges)*
cOC ratio. Circulating $\mathrm{COC}$ increased significantly after the highest MK-7 intake. Supplementation at doses below the RDA (Commission Directive 2008/100/EC; doses described as low-dose MK-7) had no significant effects on the circulating levels of both Gla-proteins.

\section{Correlations}

At baseline, significant correlations were found between sex and COC concentrations $(r-0.42, P=0.005)$. In addition, age correlated significantly with ucOC concentrations $(r-0.36, P=0.020)$ and the ucOC:COC ratio $(r-0.34$, $P=0.026)$. At baseline, a significant correlation was also found between sex and ETP values $(r 0 \cdot 40, P=0 \cdot 008)$.

Circulating MK-7 concentrations measured at the end of the intervention period negatively correlated with changes in ucOC $(r-0.37, P=0.017)$, ucOC:cOC ratio $(r-0.45$, $P=0.003)$ or dp-ucMGP concentrations $(r-0.63, P<0.001)$. End plasma phylloquinone concentrations did not correlate with changes in ucOC, ucOC:cOC ratio or dp-ucMGP concentrations.

ETP and PH values highly correlated both at baseline $(r 0 \cdot 88$ $P<0 \cdot 001)$ and at the end of the intervention $(r 0 \cdot 91, P<0 \cdot 001)$

\section{Thrombin generation}

When comparing baseline and end values, no differences were found for both ETP $(P=0 \cdot 691)$ and PH $(P=0 \cdot 844)$ values

Table 3. Circulating osteocalcin (OC) and matrix Gla-protein (MGP) at baseline and after 3-month

\begin{tabular}{|c|c|c|c|c|c|c|}
\hline \multirow[b]{2}{*}{ MK-7 dose $(\mu \mathrm{g} / \mathrm{d})$} & \multicolumn{2}{|c|}{ ucOC (ng/ml) } & \multicolumn{2}{|c|}{$\mathrm{cOC}(\mathrm{ng} / \mathrm{ml})$} & \multicolumn{2}{|c|}{ dp-ucMGP (pmol/l) } \\
\hline & Median & Range & Median & Range & Median & Range \\
\hline \multicolumn{7}{|l|}{0} \\
\hline Baseline & 3.9 & $2 \cdot 1-6 \cdot 9$ & $4 \cdot 7$ & $3 \cdot 0-8 \cdot 5$ & 435 & $203-702$ \\
\hline End & 3.7 & $1 \cdot 8-10 \cdot 1$ & $5 \cdot 2$ & $2 \cdot 2-8 \cdot 3$ & 508 & $271-841$ \\
\hline \multicolumn{7}{|l|}{10} \\
\hline Baseline & $2 \cdot 3$ & $0.7-3.4$ & 5.5 & $3 \cdot 1-10 \cdot 2$ & 471 & $166-565$ \\
\hline End & $3 \cdot 1$ & $0.9-5.9$ & 5.0 & $2 \cdot 9-12 \cdot 3$ & 414 & $235-846$ \\
\hline \multicolumn{7}{|l|}{20} \\
\hline Baseline & 4.7 & $2 \cdot 3-9 \cdot 0$ & $6 \cdot 0$ & $3 \cdot 2-7 \cdot 5$ & 476 & $247-621$ \\
\hline End & $5 \cdot 3$ & $1 \cdot 4-9 \cdot 2$ & $5 \cdot 5$ & $2 \cdot 7-8.9$ & 427 & $153-993$ \\
\hline \multicolumn{7}{|l|}{45} \\
\hline Baseline & $3 \cdot 0$ & $1 \cdot 4-6 \cdot 3$ & 6.5 & $2 \cdot 2-10 \cdot 9$ & 416 & $349-568$ \\
\hline End & $2 \cdot 5$ & $0.8-7.9$ & $6 \cdot 1$ & $2.9-8.4$ & 365 & $287-591$ \\
\hline \multicolumn{7}{|l|}{90} \\
\hline Baseline & $3 \cdot 8$ & $1 \cdot 1-10 \cdot 5$ & $4 \cdot 8$ & $2 \cdot 8-9 \cdot 6$ & 428 & $175-563$ \\
\hline End & $2 \cdot 7$ & $0 \cdot 7-8 \cdot 1$ & $5 \cdot 3$ & $2 \cdot 2-8.5$ & 284 & $120-383$ \\
\hline \multicolumn{7}{|l|}{180} \\
\hline Baseline & 4.5 & $2 \cdot 4-12 \cdot 4$ & $5 \cdot 5$ & $2 \cdot 4-11 \cdot 3$ & 426 & $292-697$ \\
\hline End & $2 \cdot 1$ & $0.8-9.5$ & 8.4 & $3 \cdot 4-15 \cdot 0$ & 257 & $230-397$ \\
\hline \multicolumn{7}{|l|}{360} \\
\hline Baseline & 1.9 & $0 \cdot 8-5 \cdot 3$ & 4.5 & $2 \cdot 0-6 \cdot 2$ & 389 & $272-516$ \\
\hline End & 0.7 & $0 \cdot 3-2 \cdot 2$ & $6 \cdot 7$ & $3.5-13.8$ & 171 & $144-198$ \\
\hline
\end{tabular}

MK-7, menaquinone-7; ucOC, uncarboxylated osteocalcin; cOC, carboxylated osteocalcin; dp-ucMGP, desphosphouncarboxylated MGP.

*A total of forty-two participants were randomised into seven groups (six subjects/group) to receive one of the following supplements (capsules): placebo or MK-7 at a daily dose of $10,20,45,90,180$ or $360 \mu \mathrm{g}$. Circulating $\mathrm{OC}$ and MGP were measured at baseline and at the end of the intervention period. 


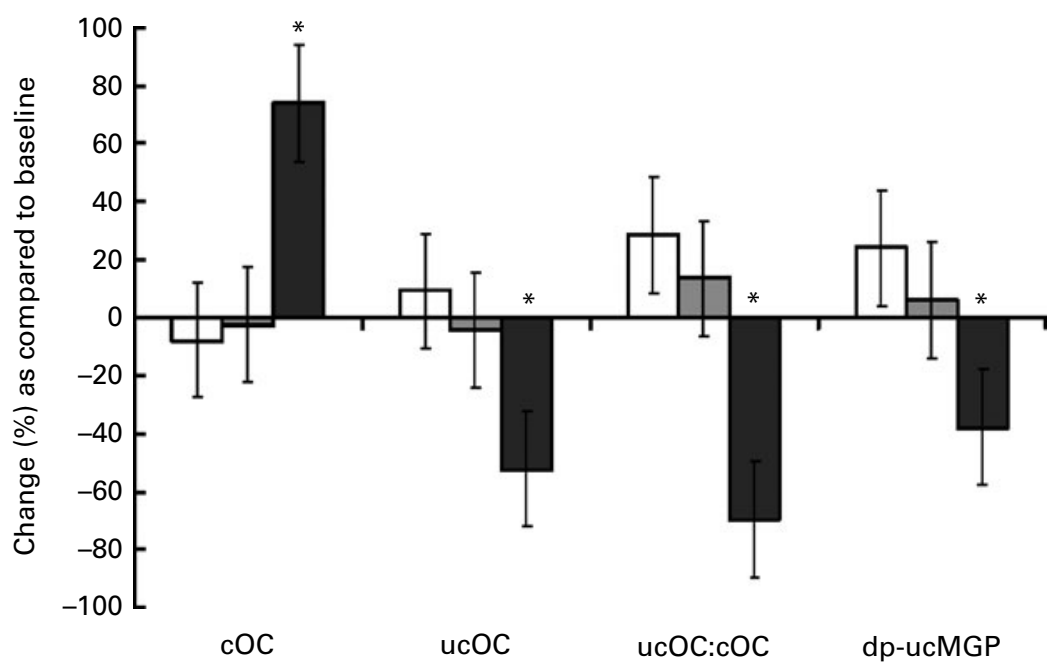

Fig. 1. A total of forty-two participants were randomised into seven groups (six subjects/group) to receive: placebo capsules or menaquinone-7 (MK-7) capsules at a daily dose of $10,20,45,90,180$ or $360 \mu \mathrm{g}$. Circulating osteocalcin (OC) and matrix Gla-protein (MGP) were measured at baseline and at the end of the intervention period. To increase the statistical power, we collapsed the seven treatment groups into three dosage groups: placebo, low-dose MK-7 (doses below the RDA, Commission Directive 2008/100/EC) and high-dose MK-7 (doses around the RDA, Commission Directive 2008/100/EC). Associations between extra MK-7 intake and the end value of the measure of interest (uncarboxylated osteocalcin (ucOC), carboxylated osteocalcin (cOC), ucOC:cOC, desphospho-uncarboxylated matrix Gla-protein (dp-ucMGP)) were investigated with ANCOVA including sex as a fixed factor and the following variables as covariates: baseline value, age and BMI. Multiple comparisons were made using Bonferroni adjustment. Mean percentage changes as compared to baseline are illustrated for the compiled groups:

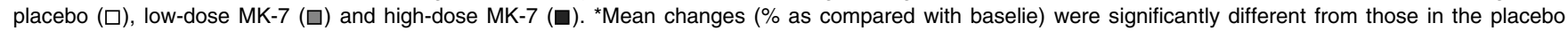
group $(P<0.05)$.

\section{Discussion}

There is some concern that current recommendations for vitamin $\mathrm{K}$ intake may be insufficient to ensure adequate function of vitamin K-dependent proteins not involved in coagulation ${ }^{(6)}$. While prothrombin is essentially $100 \%$ carboxylated under normal conditions, $10-40 \%$ of circulating OC and MGP remains undercarboxylated ${ }^{(6,9,11)}$. In line with this, circulating concentrations of ucOC and dp-ucMGP were detectable in all our volunteers. On the other hand, relatively low doses of MK-7 were sufficient to substantially increase carboxylation. A key question, of course, is whether increased extra-hepatic carboxylation contributes to better health. McCann \& Ames recently postulated that at suboptimal supply, vitamins are primarily utilised for functions required for short-term survival ${ }^{(\sigma)}$. Long-term vitamin insufficiencies may increase the development of age-related diseases, including osteoporosis, CVD and cancer. In case of vitamin $\mathrm{K}$, bleeding is the most immediate threat; so a transport system has evolved ensuring preferential targeting to the liver. This would explain why the first signs of vitamin $\mathrm{K}$ insufficiency are seen as incomplete carboxylation of the extra-hepatic Gla-proteins.

Thus far, only two dose-response studies have been performed with MK-7; one study involved reinforced natto intake and the other studied the effects of enriched olive oil. Tsukamoto et al. ${ }^{(12)}$ showed that 1-week consumption of natto containing $0.8,1.3$ or $1.8 \mathrm{mg}$ MK- $7 / 100 \mathrm{~g}$ natto increased circulating MK-7 concentrations dose-dependently in eight healthy Japanese volunteers ${ }^{(12)}$. However, only the highest dose significantly decreased serum ucOC concentrations. Circulating $\mathrm{COC}$ increased in the two highest dosing groups. It is possible that their regular natto consumption overran the benefits of additional natto intake. Recently, Brugè et al. ${ }^{(13)}$ showed in twelve healthy men and women that 2-week administration of olive oil enriched with 0,45 or $90 \mu \mathrm{g}$ MK-7 resulted in a dose-dependent increase in plasma concentrations. Only the highest dose significantly improved the carboxylation of OC. The lack of effect in both studies may reflect the small sample size and/or the short intervention period. Despite the dose-dependent MK-7 increase in our study, the lower nutritional doses $(\leq 45 \mu \mathrm{g})$ had also no significant effects on carboxylation. In agreement, a much larger sample size is probably needed to detect significant improvements after administrating such low dosages.

Further, extra menaquinone intake in the form of MK-7 supplements has been studied to date in two intervention trials; one in healthy adults ${ }^{(7)}$ and the other in healthy children ${ }^{(14)}$. Efficacy of MK-7 $(0.22 \mu \mathrm{mol})$ was compared with that of phylloquinone $(0.22 \mu \mathrm{mol})$ in eighteen healthy adults ${ }^{(7)}$. MK-7 accumulated during the first 2 weeks to reach a plateau value; phylloquinone remained slightly above baseline values. Within $3 \mathrm{~d}$, both vitamins had increased OC carboxylation, but only during MK-7 intake the improvement continued during the entire 6-week period. Thus, higher and more stable plasma concentrations were reached with MK-7, and MK-7 was more effective in carboxylating OC. Preferential tissue targeting may explain the differences in extra-hepatic degree of carboxylation. In children, 8-week supplementation with $45 \mu \mathrm{g}$ MK-7 significantly decreased circulating ucOC and the ucOC: $\mathrm{COC}$ ratio, demonstrating an improved vitamin $\mathrm{K}$ status ${ }^{(14)}$. Serum $\mathrm{COC}$ concentrations increased by $11 \%$ as compared to the placebo group; the change was however not statistically significant. The dosage of $45 \mu \mathrm{g}$ was based on the results of the adult study ${ }^{(7)}$; a dosage of $0.22 \mu \mathrm{mol}$, 
i.e. $150 \mu \mathrm{g}$ MK-7 was used for adults with an estimated weight of $80 \mathrm{~kg}$. This adult dosage was recalculated to the body weight of children (aged 6-10 years; estimated average weight $25 \mathrm{~kg}$ ), leading to a dosage of $45 \mu \mathrm{g}$ MK-7 for children.

Given the large inter-individual variability in MK-7 absorption and in effect size, we studied the association of plasma MK-7 concentrations with changes in circulating extra-hepatic Gla-proteins. Significant associations were found; the higher the end plasma MK-7 values, the larger the decrease in circulating ucOC and dp-ucMGP. Tsukamoto $e a l^{(12)}$ found a positive relationship between serum MK-7 and COC concentrations, both in men and in women. For this experiment, both markers were measured in 134 young adults who were classified according to their intake of dietary natto: rare, occasional and frequent. In agreement, Brugè et $a l .{ }^{(13)}$ found a significant correlation between differences in plasma MK-7 and changes in the cOC:ucOC ratio. Additionally, a high intra- and interindividual biological variability in the cOC:ucOC ratio was described. Considering the high variability of this index, older volunteers were postulated to show more consistent effects due to a higher requirement of vitamin $\mathrm{K}$.

To investigate the potential effects of vitamin K supplements on coagulation, we have used baseline and end-point samples to compare the paired ETP curves generated by CAT. CAT is a more sensitive assay than the routine coagulation assays for anticoagulation monitoring and reversal with different heparinoids $^{(15)}$. Using this highly sensitive system, no effect on the blood coagulation system was observed. On a theoretical basis such an effect is not to be expected, because in healthy subjects all clotting factors contain the maximum number of Gla-residues (e.g. ten for prothrombin, twelve for factor IX). Incompletely carboxylated coagulation factors are seldom seen in the healthy population, but only in patients on coumarin derivates or with malabsorption of fat-soluble vitamins. In the wide range of MK-7 intake of the present study, no effect on the blood coagulation system was observed.

In conclusion, daily intake of MK-7 at doses in the order of the RDA (Commission Directive 2008/100/EC) results in measurable changes in circulating ucOC and ucMGP. Given the fact that in the Western society the total vitamin $\mathrm{K}$ intake amounts to $100-150 \mu \mathrm{g} / \mathrm{d}$, and that the MK-7 was given on top of a regular diet, this is remarkably low and demonstrates the high potency of this vitamer. MK-7 is safe with respect to the haemostatic system, i.e. in subjects not on oral anticoagulants, it does not increase the thrombosis risk even at doses above the RDA (Commission Directive 2008/100/EC).

\section{Acknowledgements}

The present study was supported by Nattopharma ASA (Oslo, Norway). None of the authors had any possible conflicts of interest. The contributions of the authors to this study were as follows: C. V. and L. J. S. designed the research; M. H. K., E. J. M. and K. J. T. conducted the research (M. H. K. and K. J. T. were responsible for the daily management of the study, E. J. M. was responsible for the OC and MGP analyses); E. T. and E. C. C. analysed the data; E. T., E. S. and C. V. wrote the paper; C. V. had primary responsibility for the final content. All authors read and approved the final manuscript.

\section{References}

1. Shearer MJ \& Newman P (2008) Metabolism and cell biology of vitamin K. Thromb Haemost 100, 530-547.

2. Burstyn-Cohen T, Heeb MJ \& Lemke G (2009) Lack of protein $S$ in mice causes embryonic lethal coagulopathy and vascular dysgenesis. J Clin Invest 119, 2942-2953.

3. Szulc P, Chapuy MC, Meunier PJ, et al. (1993) Serum undercarboxylated osteocalcin is a marker of the risk of hip fracture in elderly women. J Clin Invest 91, 1769-1774.

4. Ueland T, Gullestad L, Dahl CP, et al. (2010) Undercarboxylated matrix Gla protein is associated with indices of heart failure and mortality in symptomatic aortic stenosis. J Intern Med 268, 483-492.

5. Nimptsch K, Rohrmann S, Kaaks R, et al. (2010) Dietary vitamin $\mathrm{K}$ intake in relation to cancer incidence and mortality: results from the Heidelberg cohort of the European Prospective Investigation into Cancer and Nutrition (EPIC-Heidelberg). Am J Clin Nutr 91, 1348-1358.

6. McCann JC \& Ames BN (2009) Vitamin K, an example of triage theory: is micronutrient inadequacy linked to diseases of aging? Am J Clin Nutr 90, 889-907.

7. Schurgers LJ, Teunissen KJ, Hamulyak K, et al. (2007) Vitamin K-containing dietary supplements: comparison of synthetic vitamin $\mathrm{K}_{1}$ and natto-derived menaquinone-7. Blood 109, 3279-3283.

8. Schurgers LJ \& Vermeer C (2000) Determination of phylloquinone and menaquinones in food. Effect of food matrix on circulating vitamin $\mathrm{K}$ concentrations. Haemostasis $\mathbf{3 0}$, 298-307.

9. Cranenburg EC, Koos R, Schurgers LJ, et al. (2010) Characterisation and potential diagnostic value of circulating matrix Gla protein (MGP) species. Thromb Haemost 104, 811-822.

10. Hemker HC, Giesen P, AlDieri R, et al. (2002) The calibrated automated thrombogram (CAT): a universal routine test for hyper- and hypocoagulability. Pathophysiol Haemost Thromb 32, 249-253.

11. Booth SL, Dallal G, Shea MK, et al. (2008) Effect of vitamin K supplementation on bone loss in elderly men and women. J Clin Endocrinol Metab 93, 1217-1223.

12. Tsukamoto $\mathrm{Y}$, Ichise $\mathrm{H}$, Kakuda $\mathrm{H}$, et al. (2000) Intake of fermented soybean (natto) increases circulating vitamin $\mathrm{K}_{2}$ (menaquinone-7) and gamma-carboxylated osteocalcin concentration in normal individuals [In Process Citation]. J Bone Miner Metab 18, 216-222.

13. Brugè F, Bacchetti T, Principi F, et al. (2011) Olive oil supplemented with menaquinone-7 significantly affects osteocalcin carboxylation. Br J Nutr 106, 1058-1062.

14. van Summeren MJ, Braam LA, Lilien MR, et al. (2009) The effect of menaquinone-7 (vitamin $\mathrm{K}_{2}$ ) supplementation on osteocalcin carboxylation in healthy prepubertal children. Br J Nutr 102, 1171-1178.

15. Gatt A, Riddell A, van Veen JJ, et al. (2009) Optimizing warfarin reversal - an ex vivo study. J Thromb Haemost 7 , $1123-1127$. 